PROCEEDINGS OF THE

AMERICAN MATHEMATICAL SOCIETY

Volume 132, Number 4, Pages 965-972

S 0002-9939(03)07181-8

Article electronically published on July 7, 2003

\title{
A QUASI-HOPF ALGEBRA FREENESS THEOREM
}

\author{
PETER SCHAUENBURG
}

(Communicated by Martin Lorenz)

\begin{abstract}
We prove the quasi-Hopf algebra version of the Nichols-Zoeller theorem: A finite dimensional quasi-Hopf algebra is free over any quasi-Hopf subalgebra.
\end{abstract}

\section{INTRODUCTION}

The first of Kaplansky's conjectures on Hopf algebras [5 asked whether a Hopf algebra $H$ is always free over any Hopf subalgebra $K$. While this is false in the infinite-dimensional case, as shown by Oberst and Schneider [11], it is true when $H$ is finite dimensional. This is the content of the celebrated Nichols-Zoeller theorem 10]. More generally, Nichols and Zoeller prove that Hopf modules in the category $\mathcal{M}_{K}^{H}$ are always free as $K$-modules, provided again that $H$ is a finite-dimensional Hopf algebra and $K$ a Hopf subalgebra. The Nichols-Zoeller theorem is an indispensable tool in the study of finite-dimensional Hopf algebras.

In the present paper, we will prove essentially the same results for quasi-Hopf algebras in place of Hopf algebras. Quasi-Hopf algebras were introduced by Drinfeld [1. By definition, a quasibialgebra $H$ is an algebra and a non-coassociative coalgebra, whose lack of coassociativity is controlled by an invertible element $\phi \in$ $H \otimes H \otimes H$, the associator; more precisely, comultiplication is coassociative up to conjugation by $\phi$. The "meaning" of the definition is already explained in Drinfeld's paper: The axioms are such that the category ${ }_{H} \mathcal{M}$ of modules over $H$ is a monoidal category with respect to the diagonal module structure on the tensor product of $H$-modules, taken over the base field $k$. The rather complicated axioms for a quasi-antipode are designed so that one can define a dual $H$-module for any finite-dimensional $H$-module.

The question for a Nichols-Zoeller theorem for quasi-Hopf algebras was brought to the author's attention by Robert Fischer. We shall prove that a finite-dimensional quasi-Hopf algebra $H$ is a free module over any quasi-Hopf subalgebra $K$. This is by definition a subspace $K \subset H$ that has a quasi-Hopf algebra structure for which the inclusion is a quasi-Hopf algebra map; in particular, $K \otimes K \otimes K$ should contain the associator $\phi$ of $H$. Interesting examples of quasi-Hopf algebra inclusions in this sense have recently been studied (in dual form) by Masuoka [7], 8]. We note that in Masuoka's examples, which are generalizations of bicrossproduct constructions,

Received by the editors March 1, 2002 and, in revised form, November 24, 2002.

2000 Mathematics Subject Classification. Primary 16W30.

Key words and phrases. Quasi-Hopf algebra, Nichols-Zoeller theorem, Hopf module.

(C)2003 American Mathematical Society 
$H$ is a free $K$-module by construction. The same is true for the embedding of a finite-dimensional quasi-Hopf algebra in its Drinfeld double; cf. [2], 3].

We will also prove a quasi-Hopf version of the freeness result of Nichols and Zoeller on Hopf modules. However, we will not use this result to prove freeness of $H$ over $K$. In fact, the freeness of $H$ over $K$ will be proved in the more general situation that $K$ is a subalgebra and (non-coassociative) subcoalgebra of $H$, which has some structure of quasi-Hopf algebra with its own associator that needs not coincide with that of $H$. Natural examples of such a situation come immediately to mind: If $K$ and $F$ are quasi-Hopf algebras, then $K \otimes F:=H$ is a quasi-Hopf algebra, containing $K$ as a subalgebra and subcoalgebra, but with its associator not contained in $K \otimes K \otimes K$, unless the associator of $F$ is trivial. (Incidentally, $H$ is of course a free $K$-module in this situation.)

Our proofs will follow the original proofs of Nichols and Zoeller, or more precisely the account in Montgomery's book [9, quite closely. A lemma on modules over Frobenius algebras, in particular, will retain its key rôle in the proof. The main obstacle for the generalization is the constant use of Hopf module categories like $\mathcal{M}_{K}^{H}$, and of structure theorems for Hopf modules. Note that the said Hopf module category is not defined when $H$ is a quasi-Hopf algebra, since then $H$ need not be a coalgebra.

These difficulties can be overcome by considering suitable Hopf module categories that have been defined by Hausser and Nill [4. The point is that while a quasibialgebra is not a coalgebra, one can still define Hopf bimodule categories ${ }_{H} \mathcal{M}_{H}^{H}$. This is surprising at first sight, but the definition by Hausser and Nill turns out to be simply the definition of an $H$-comodule - but within the monoidal category ${ }_{H} \mathcal{M}_{H}$ of $H$-bimodules, in which $H$ is a coalgebra after all. This categorical viewpoint on Hopf modules was stressed and used in [15]. Hausser and Nill also proved a structure theorem for Hopf modules in ${ }_{H} \mathcal{M}_{H}^{H}$, generalizing the well-known structure theorem for Hopf modules in $\mathcal{M}_{H}^{H}$ with an ordinary Hopf algebra $H$.

\section{QuASI-Hopf ALGEBRAS}

Throughout the paper, we work over some base field $k$. Tensor products, algebras and the like are over $k$.

We recall the definition and some basic facts concerning quasibialgebras, quasiHopf algebras, and their relation to monoidal categories. General references are Drinfeld's original paper [1, and Kassel's book [6].

A quasibialgebra $H=(H, \Delta, \varepsilon, \phi)$ consists of an algebra $H$, algebra maps $\Delta: H$ $\rightarrow H \otimes H$ and $\varepsilon: H \rightarrow k$, and an invertible element $\phi \in H^{\otimes 3}$, the associator, such that

$$
\begin{gathered}
(\varepsilon \otimes H) \Delta(h)=h=(H \otimes \varepsilon) \Delta(h), \\
(H \otimes \Delta) \Delta(h) \cdot \phi=\phi \cdot(\Delta \otimes H) \Delta(h), \\
(H \otimes H \otimes \Delta)(\phi) \cdot(\Delta \otimes H \otimes H)(\phi)=(1 \otimes \phi) \cdot(H \otimes \Delta \otimes H)(\phi) \cdot(\phi \otimes 1), \\
(H \otimes \varepsilon \otimes H)(\phi)=1
\end{gathered}
$$

hold for all $h \in H$. We will write $\Delta(h)=: h_{(1)} \otimes h_{(2)}, \phi=\phi^{(1)} \otimes \phi^{(2)} \otimes \phi^{(3)}$, and $\phi^{-1}=\phi^{(-1)} \otimes \phi^{(-2)} \otimes \phi^{(-3)}$.

If $H$ is a quasibialgebra, then the category ${ }_{H} \mathcal{M}$ of left $H$-modules is a monoidal category in the following way: For $V, W \in{ }_{H} \mathcal{M}$ the tensor product $V \otimes W$ is an 
$H$-module by $h(v \otimes w)=h_{(1)} v \otimes h_{(2)} w$. The base ring $k$ is an $H$-module via $\varepsilon$. The canonical morphisms $V \cong V \otimes k \cong k \otimes V$ are $H$-linear for $V \in{ }_{H} \mathcal{M}$. The map

$$
\Phi:(U \otimes V) \otimes W \ni u \otimes v \otimes w \mapsto \phi^{(1)} u \otimes \phi^{(2)} v \otimes \phi^{(3)} w \in U \otimes(V \otimes W)
$$

for $H$-modules $U, V, W$ is $H$-linear as a consequence of (2.2), and satisfies Mac Lane's pentagon axiom for a monoidal category as a consequence of (2.3).

We denote $H$ with the opposite multiplication, co-opposite comultiplication, or both, by $H^{\mathrm{op}}, H^{\mathrm{cop}}$, and $H^{\mathrm{bop}}$, respectively. If $(H, \phi)$ is a quasibialgebra, so are $\left(H^{\mathrm{op}}, \phi^{-1}\right),\left(H^{\mathrm{cop}}, \phi^{(3)} \otimes \phi^{(2)} \otimes \phi^{(1)}\right)$, and hence also $\left(H^{\mathrm{bop}}, \phi^{(-3)} \otimes \phi^{(-2)} \otimes \phi^{(-1)}\right)$. If $(B, \psi)$ is another quasibialgebra, then $H \otimes B$ is a quasibialgebra with associator $\phi^{(1)} \otimes \psi^{(1)} \otimes \phi^{(2)} \otimes \psi^{(2)} \otimes \phi^{(3)} \otimes \psi^{(3)}$.

A quasi-antipode $(S, \alpha, \beta)$ for a quasibialgebra $H$ consists of an algebra antiautomorphism $S$ of $H$, and elements $\alpha, \beta \in H$, such that

$$
\begin{aligned}
S\left(h_{(1)}\right) \alpha h_{(2)} & =\varepsilon(h) \alpha, & h_{(1)} \beta S\left(h_{(2)}\right) & =\varepsilon(h) \beta, \\
\phi^{(1)} \beta S\left(\phi^{(2)}\right) \alpha \phi^{(3)} & =1, & S\left(\phi^{(-1)}\right) \alpha \phi^{(-2)} \beta \phi^{(-3)} & =1
\end{aligned}
$$

hold in $H$, for $h \in H$. A quasi-Hopf algebra is a quasibialgebra with a quasiantipode. If $H$ is a quasi-Hopf algebra, then so are $H^{\mathrm{op}}, H^{\mathrm{cop}}$, and $H^{\mathrm{bop}}$; for later use we note that the underlying map of the quasi-antipode of $H^{\mathrm{op}}$ is $S^{-1}$. Let $H$ be a quasi-Hopf algebra. If $V$ is a finite-dimensional left $H$-module, then $V^{*}$, the dual vector space, becomes a left $H$-module with the action induced by the transpose of the original action of $H$ via the quasi-antipode map, that is, by setting $\langle h \cdot \varphi, v\rangle=\langle\varphi, S(h) v\rangle$, for $h \in H, \varphi \in V^{*}$, and $v \in V$, where $\langle$,$\rangle denotes$ evaluation. The $H$-module $V^{*}$ becomes a dual object to $V$ in the monoidal category of $\mathrm{H}$-modules, if we define the evaluation and co-evaluation maps

$$
\text { ev : } V^{*} \otimes V \rightarrow k \quad \text { and } \quad \mathrm{db}: k \rightarrow V \otimes V^{*}
$$

by $\operatorname{ev}(\varphi \otimes v)=\langle\varphi, \alpha v\rangle$ and $\operatorname{db}(1)=\beta v_{i} \otimes v^{i}$, where $\left(v_{i}\right)$ and $\left(v^{i}\right)$ are dual bases, with summation over $i$ understood.

Let $(H, \phi)$ be a quasibialgebra. A subquasibialgebra $K \subset H$ is a subspace with a quasibialgebra structure for which the inclusion is a quasibialgebra map. Equivalently, $K$ is a subalgebra and (non-coassociative) subcoalgebra of $H$, and $\phi \in K \otimes K \otimes K$. If $H$ is a quasi-Hopf algebra with quasi-antipode $(S, \alpha, \beta)$, and $K \subset H$ is a subquasibialgebra, one may have different opinions about when $K$ should be called a quasi-Hopf subalgebra. It will be sufficient for all purposes in this paper to assume $K$ has a quasi-antipode, without assuming that it is in any way related to the quasi-antipode of $H$ chosen, or, in fact, to any other quasi-antipode of $H$. There is an obvious stricter notion which says $K$ is a quasi-Hopf subalgebra of the quasi-Hopf algebra $(H, \phi, S, \alpha, \beta)$ if it is a subquasibialgebra closed under $S$ and containing $\alpha$ and $\beta$.

\section{Hopf MODUlES}

Let $H$ be a quasibialgebra. Then it is impossible to define a comodule over $H$, since $H$ is not a coassociative coalgebra. It is also impossible to define a Hopf module category $\mathcal{M}_{H}^{H}$ with one module and one comodule structure.

However, there is a definition of Hopf modules in ${ }_{H} \mathcal{M}_{H}^{H}$, which can be viewed as a special case of the theory of (co)algebras and (co)modules within monoidal categories. For a development of this theory, in which many of the elementary facts 
and constructions of ordinary ring theory can be carried over to quite arbitrary abstract monoidal categories replacing the category of abelian groups or $k$-modules, we refer the reader to [13] and [14]. We have applied this point of view on Hopf modules over quasi-Hopf algebras in [15]; it allows us to get away without lengthy calculations involving associators and the explicit use of the quasibialgebra axioms in many cases.

The axioms of a quasibialgebra imply that $H$ is a coassociative coalgebra within the monoidal category ${ }_{H} \mathcal{M}_{H}$ of $H$-H-bimodules: The associator morphism in ${ }_{H} \mathcal{M}_{H} \cong{ }_{H \text { op } \otimes H} \mathcal{M}$ is given by

$\Phi:(U \otimes V) \otimes W \ni u \otimes v \otimes w \mapsto \phi^{(1)} u \phi^{(-1)} \otimes \phi^{(2)} v \phi^{(-2)} \otimes \phi^{(3)} w \phi^{(-3)} \in U \otimes(V \otimes W)$, so that coassociativity of $H$ as a coalgebra in ${ }_{H} \mathcal{M}_{H}$ is, in fact, the same as (2.2).

Now one can define Hopf modules (which other authors prefer to call quasi-Hopf modules) over $H$, as first introduced by Hausser and Nill 4], as follows:

Definition 3.1. Let $H$ be a quasibialgebra. A Hopf module $M \in{ }_{H} \mathcal{M}_{H}^{H}$ is a right $H$-comodule within the monoidal category ${ }_{H} \mathcal{M}_{H}$ of $H$-bimodules. Explicitly, $M$ is an $H$-H-bimodule equipped with a map $\delta: M \rightarrow M \otimes H, \delta(m)=m_{(0)} \otimes m_{(1)}$, satisfying $m_{(0)} \varepsilon\left(m_{(1)}\right)=m$ and

$$
(M \otimes \Delta) \delta(m) \cdot \phi=\phi \cdot(\delta \otimes H) \delta(m)
$$

for all $m \in M$, where the multiplication by $\phi$ now takes place in the $H \otimes H \otimes H$ bimodule $M \otimes H \otimes H$.

More generally, let $K \subset H$ be a subquasibialgebra. Then $H$ is also a coalgebra in ${ }_{K} \mathcal{M}_{K}$, and we define the Hopf module category ${ }_{K} \mathcal{M}_{K}^{H}$ to be the category of $H$-comodules within ${ }_{K} \mathcal{M}_{K}$.

We note that Hopf module categories like ${ }_{K}^{H} \mathcal{M}_{K},{ }_{K}^{H} \mathcal{M}_{K}^{H}$ can be defined in the same way.

The interpretation of Hopf modules as comodules within some monoidal category gives an immediate supply of constructions of and with such objects. We refer the reader to 15 for examples rather than giving explicit details on the following uses of the general principle:

Remark 3.2. Let $H$ be a quasibialgebra and $K$ a quasi-Hopf subalgebra.

(1) Whenever $P \in{ }_{K} \mathcal{M}_{K}$, and $M \in{ }_{K} \mathcal{M}_{K}^{H}$, then $. P . \otimes . M: \in_{K} \mathcal{M}_{K}^{H}$. Here and below the dots are supposed to indicate that $P \otimes M$ is endowed with the diagonal left and right $K$-module structures, and the right $H$-comodule structure induced by that of $M$, but taken within the monoidal category ${ }_{K} \mathcal{M}_{K}$ with its nontrivial associator isomorphism. Explicitly, this means that the comodule structure is given by

$$
\begin{aligned}
P \otimes M & \rightarrow(P \otimes M) \otimes H, \\
p \otimes m & \mapsto \phi^{(-1)} p \phi^{(1)} \otimes \phi^{(-2)} m_{(0)} \phi^{(2)} \otimes \phi^{(-3)} m_{(1)} \phi^{(3)} .
\end{aligned}
$$

(2) As a special case, for any $P \in{ }_{K} \mathcal{M}_{K}$ we have $. P . \otimes . H: \in_{K} \mathcal{M}_{K}^{H}$. This is the cofree right $H$-comodule generated by $P$ within the monoidal category ${ }_{K} \mathcal{M}_{K}$

(3) Again as a special case, one can consider $. V \otimes . H: \in{ }_{K} \mathcal{M}_{K}^{H}$ for any left module $V \in{ }_{K} \mathcal{M}$. 
(4) As in any monoidal category, there is a notion of cotensor product of a right and a left $H$-comodule within the category ${ }_{K} \mathcal{M}_{K}$, that is, a cotensor product $M \square_{H} N \in{ }_{K} \mathcal{M}_{K}$ for $M \in{ }_{K} \mathcal{M}_{K}^{H}$ and $N \in{ }_{K}^{H} \mathcal{M}_{K}$. About this construction, we shall only need to know that

$$
M \underset{H}{\square}(: H . \otimes . P .) \cong M \otimes P
$$

as $K$-K-bimodules, for any $M \in{ }_{K} \mathcal{M}_{K}^{H}$ and $P \in{ }_{K} \mathcal{M}_{K}$. Here, $: H . \otimes . P . \in$ ${ }_{K}^{H} \mathcal{M}_{K}$ is a left-right switched version of the construction in (2).

The structure theorem for Hopf modules over quasi-Hopf algebras proved by Hausser and Nill [4, Prop.3.11] says that, for a quasi-Hopf algebra $H$, the Hopf modules in ${ }_{H} \mathcal{M}_{H}^{H}$ constructed in part (3) of Remark 3.2 are the only ones there are:

Theorem 3.3. Let $H$ be a quasi-Hopf algebra. Then the functor

$$
{ }_{H} \mathcal{M} \ni V \mapsto . V \otimes . H: \in{ }_{H} \mathcal{M}_{H}^{H}
$$

is a category equivalence. In particular, every Hopf module in ${ }_{H} \mathcal{M}_{H}^{H}$ is a free right $H$-module.

We take the liberty to refer to 15] for another proof.

The main application of Hopf module theory in [4] is the development of an integral theory for quasi-Hopf algebras. In particular, Hausser and Nill 4 , Thm. 4.3] show the existence of a cointegral $\lambda \in H^{*}$ for any finite-dimensional quasi-Hopf algebra $H$, which is nondegenerate and hence makes $H$ a Frobenius algebra. Since the fact that $H$ is Frobenius is of key importance for the Nichols-Zoeller result, we shall sketch a short proof of this fact for completeness:

Theorem 3.4. Let $H$ be a finite-dimensional quasi-Hopf algebra. Then there exists a nondegenerate linear form $\lambda \in H^{*}$, so that $H$ is a Frobenius algebra.

(Nondegeneracy means that $H \times H \ni(g, h) \mapsto \lambda(g h) \in k$ is a nondegenerate bilinear form.)

Proof. Since $H$ is a quasi-Hopf algebra, so is $H^{\mathrm{op}}$, and hence $H^{\mathrm{op}} \otimes H$. This implies that for each $V \in{ }_{H} \mathcal{M}_{H} \cong H^{\mathrm{op} \otimes H} \mathcal{M}$ the dual space $V^{*}$ becomes a dual object for $V$ within the monoidal category ${ }_{H} \mathcal{M}_{H}$, with module structures induced by the antipode of $H^{\mathrm{op}} \otimes H$. More precisely, the $H$ - $H$-bimodule structure on $V^{*}$ is given by

$$
\langle g \cdot \varphi \cdot h, v\rangle=\left\langle\varphi, S(g) v S^{-1}(h)\right\rangle
$$

for $v \in V, \varphi \in V^{*}$, and $g, h \in H$, where $\langle$,$\rangle denotes evaluation. It is easy to check$ (and a very special case of [13, Cor. 3.7]) that when an object $V$ of a monoidal category $\mathcal{C}$ is a left comodule over some coalgebra $C \in \mathcal{C}$, and has a dual $V^{*}$, then $V^{*}$ is a right $C$-comodule. For example, since $H$ is a left $H$-comodule within the monoidal category ${ }_{H} \mathcal{M}_{H}$, its dual $H^{*}$ is a right $H$-comodule (whose structure map we will not need to know), that is, an object of ${ }_{H} \mathcal{M}_{H}^{H}$. It follows from Theorem 3.3 that there is a (one-dimensional) left $H$-module $V$ and an isomorphism

$$
\psi: . V \otimes . H: \rightarrow H^{*}
$$

of Hopf modules in ${ }_{H} \mathcal{M}_{H}^{H}$. Since $\psi$ is a right $H$-module map, it has the form $\psi(v \otimes h)=\psi_{0}(v) h$ for some $\psi_{0}: V \rightarrow H^{*}$. Let $v \in V \backslash\{0\}$, and set $\lambda:=\psi_{0}(v)$. 
Then it follows that

$$
H \ni h \mapsto\left(\lambda \cdot h: g \mapsto \lambda\left(g S^{-1}(h)\right)\right) \in H^{*}
$$

is an isomorphism. Therefore, $\lambda$ is nondegenerate and $H$ is Frobenius.

\section{NichOLS-ZOelleR FOR QUASI-Hopf ALGEBRAS}

In this section we prove the quasi-Hopf algebra version of the Nichols-Zoeller theorem. The proof follows quite precisely the course taken in the ordinary Hopf case, where we rely on the account given in Montgomery's book [9]. However, one has to take special care whenever a Hopf module argument comes in, due to the fact that Hopf modules have a delicate definition. In particular, the relevant Hopf module categories used in the original proof do not exist in the quasi-Hopf case; luckily, the actual objects in consideration can be located in Hopf module categories which do exist.

Theorem 4.1. Let $K$ be a finite-dimensional quasi-Hopf algebra, and $W$ a finitely generated right $K$-module. Suppose there exists a finitely generated faithful right $K$-module $V$ such that $W \otimes V \cong W^{\operatorname{dim} V}$ as right $K$-modules. Then $W$ is free over $K$.

Proof. Since $K$ is Frobenius, we can apply [9, Prop. 3.1.2] which tells us that it is enough to show that some power of $W$ is free. Also, we can apply [9, Lem. 3.1.1] to conclude that there is $r>0$ such that $W^{r} \cong F \oplus E$ with $F$ a free $K$-module and $E$ a $K$-module that is not faithful, and also there is $s>0$ such that $V^{s} \cong F^{\prime} \oplus E^{\prime}$ with $F^{\prime}$ free and $E^{\prime}$ not faithful. Since $W^{r} \otimes V^{s} \cong(W \otimes V)^{r s} \cong W^{r s} \operatorname{dim} V$, we can replace $W$ by $W^{r}$ and $V$ by $V^{s}$, and assume that $W \cong F \oplus E$ and $V \cong F^{\prime} \oplus E^{\prime}$.

So far, everything proceeded as in $[9]$. Now we consider $: K . \otimes . V \in{ }_{K}^{K} \mathcal{M}_{K}$ by applying part (3) of Remark 3.2 to $K^{\text {bop }}$. By the version of Theorem 3.3 for $K^{\text {bop }}$, this is a free left $K$-module, hence $. K \otimes . V \cong K^{t}$ as left $K$-modules, where $t:=\operatorname{dim} V$. Since $F$ is free, it follows that $F \otimes V \cong F^{t}$. Now

$$
F^{t} \oplus E^{t} \cong W^{t} \cong W \otimes V \cong(F \otimes V) \oplus(E \otimes V) \cong F^{t} \oplus(E \otimes V),
$$

and the Krull-Schmidt theorem implies

$$
E^{t} \cong E \otimes V \cong E \otimes\left(F^{\prime} \oplus E^{\prime}\right) \cong\left(E \otimes F^{\prime}\right) \oplus\left(E \otimes E^{\prime}\right) .
$$

Since $E$ is not faithful, neither is $E^{t}$, and hence neither is $E \otimes F^{\prime}$. But $E \otimes F^{\prime}$ is free, which finally implies that $E=0$ and hence $W$ is free. To see that $E \otimes F^{\prime}$ is free, it is in turn sufficient to see that $E \otimes K$ is free, which follows from Theorem 3.3, since we can consider $E . \otimes . K: \in{ }_{K} \mathcal{M}_{K}^{K}$ by part (3) of Remark 3.2

We arrive immediately at the quasi-Hopf version of the Nichols-Zoeller theorem, for the more general situation advertised in the introduction.

Theorem 4.2. Let $H$ be a finite-dimensional quasi-Hopf algebra, and let $K \subset H$ be a subalgebra and (non-coassociative) subcoalgebra that admits a quasi-Hopf algebra structure. Then $H$ is a free (say, right) $K$-module.

Proof. Consider the faithful right $K$-module $H$. By Theorem 4.1 it suffices to show that $H \otimes H \cong H^{\operatorname{dim} H}$ as right $K$-module. Since $K$ is a subcoalgebra of $H$, the $K$-module $H \otimes H$ with diagonal module structure is the same as the restriction to $K$ of the $H$-module $H \otimes H$ with diagonal module structure. But $H . \otimes . H: \in{ }_{H} \mathcal{M}_{H}^{H}$ by 
part (2) of Remark 3.2, so that we can apply Theorem 3.3) to conclude that $H \otimes H$ is indeed a free right $H$-module, and $H \otimes H \cong H^{\operatorname{dim} H}$ even as $H$-modules.

To prepare for an application to semisimple quasi-Hopf algebras, modelled on [9, Cor. 3.2.3], we will prove a result of Panaite characterizing semisimple quasiHopf algebras in terms of integrals; our proof will utilize Hopf modules.

Theorem 4.3 (Panaite 12]). A finite-dimensional quasi-Hopf algebra $H$ is semisimple if and only if it contains a left integral $t \in H$ with $\varepsilon(t) \neq 0$ (one might then as well assume that $\varepsilon(t)=1)$. Here, an integral is an element satisfying $h t=\varepsilon(h) t$ for all $h \in H$.

Proof. If $H$ is semisimple, the epimorphism $\varepsilon: H \rightarrow k$ of left $H$-modules splits, which is the same as the existence of an integral $t$ with $\varepsilon(t)=1$.

On the other hand, assume that $\varepsilon: H \rightarrow k$ splits as an $H$-module map. Then for any $V \in{ }_{H} \mathcal{M}$ the epimorphism $H \otimes V \stackrel{\varepsilon \otimes V}{\longrightarrow} V$ splits. $: H . \otimes . V \in{ }_{H}^{H} \mathcal{M}_{H}$ is a Hopf module, hence a free left module by Theorem 3.3, which shows that $V$ is projective.

Corollary 4.4. Let $H$ be a finite-dimensional semisimple quasi-Hopf algebra. Let $K \subset H$ be a subalgebra and (non-coassociative) subcoalgebra that has an associator making it a quasi-Hopf algebra.

Then $K$ is also semisimple.

Proof. By Theorem $4.3 H$ contains a left integral $t$ with $\varepsilon(t) \neq 0$. Since $H$ is a free left $K$-module, we can copy word-by-word the proof of part 2 of [9, Lemma 2.2.2] and conclude that $K$ also contains such an integral. By Theorem 4.3 again $K$ is semisimple.

We now turn to the more general version of the original Nichols-Zoeller theorem stating that every Hopf module in $\mathcal{M}_{K}^{H}$ is free as a $K$-module. We shall show the same for such objects in ${ }_{K} \mathcal{M}_{K}^{H}$ that are finitely generated $K$-modules. In order that the indicated Hopf modules be defined in the first place, it will be necessary to assume that $K$ is a subquasibialgebra of $H$. The weaker assumption in Theorem 4.2 would not be sufficient for this purpose.

Theorem 4.5. Let $H$ be a finite-dimensional quasi-Hopf algebra and $K \subset H a$ subquasibialgebra which has a quasi-antipode. Then every Hopf module $M \in{ }_{K} \mathcal{M}_{K}^{H}$ that is finitely generated as a $K$-module, is free as a left $K$-module.

Proof. By part (2) of Remark 3.2 we can consider :H. $\otimes . H . \in{ }_{H}^{H} \mathcal{M}_{H}$, and by Theorem 3.3 it follows that $: H . \otimes . H . \cong: H . \otimes V$. for some right $H$-module $V$. (In fact, $V=H_{\text {ad }}$, which is of no importance here.)

Now we consider $: H . \otimes . H . \in{ }_{K}^{H} \mathcal{M}_{K}$ instead, where we still have the same isomorphism, of course. We conclude that, calculating with cotensor product within the monoidal category ${ }_{K} \mathcal{M}_{K}$ as indicated in part (4) of Remark [3.2,

$$
\begin{aligned}
. M . \otimes . H . & \cong . M:{ }_{H}(: H . \otimes . H .) \\
& \cong M \underset{H}{\square}(: H . \otimes V .) \\
& \cong . M . \otimes V .
\end{aligned}
$$

Forgetting about the right module structure, we conclude $M \otimes H \cong M^{\operatorname{dim} V}$ as left $K$-modules, and apply Theorem 4.1 to conclude that $M$ is a free $K$-module. 


\section{REFERENCES}

[1] V. G. Drinfel'd, Quasi-Hopf algebras, Leningrad Math. J. 1 (1990), 1419-1457. MR 91b:17016

[2] Frank Hausser and Florian Nill, Diagonal crossed products by duals of quasi-quantum groups, Rev. Math. Phys. 11 (1999), 553-629. MR 2000d:81069

[3] _ Doubles of quasi-quantum groups, Comm. Math. Phys. 199 (1999), 547-589. MR 2000a: 16075

[4] Integral theory for quasi-Hopf algebras, preprint (math.QA/9904164).

[5] Irving Kaplansky, Bialgebras, University of Chicago Lecture Notes, 1975. MR [55:8087

[6] Christian Kassel, Quantum groups, Graduate Texts in Math., vol. 155, Springer-Verlag, 1995. MR 96e:17041

[7] Akira Masuoka, Hopf algebra extensions and cohomology, New directions in Hopf algebras, Math. Sci. Res. Inst. Publ., vol. 43, Cambridge Univ. Press, Cambridge, 2002, pp. 167-209. MR 2003d: 16050

[8] Akira Masuoka, Cohomology and coquasi-bialgebra extensions associated to a matched pair of bialgebras, Adv. Math. 173 (2003), 262-315.

[9] Susan Montgomery, Hopf algebras and their actions on rings, CBMS Regional Conference Series in Mathematics, vol. 82, Amer. Math. Soc., Providence, Rhode Island, 1993. MR 94i:16019

[10] Warren D. Nichols and M. B. Zoeller, A Hopf algebra freeness theorem, Amer. J. Math. 111 (1989), 381-385. MR 90c: 16008

[11] U. Oberst and H.-J. Schneider, Untergruppen formeller Gruppen von endlichem Index, J. Algebra 31 (1974), 10-44. MR 50:13057

[12] Florin Panaite, A Maschke-type theorem for quasi-Hopf algebras, Rings, Hopf algebras, and Brauer groups (Antwerp/Brussels, 1996), Marcel Dekker, New York, 1998, pp. 201-207. MR 99k:16085

[13] Bodo Pareigis, Non-additive ring and module theory I. General theory of monoids, Publ. Math. Debrecen 24 (1977), 189-204. MR 56:8656

[14] $\ldots$ Non-additive ring and module theory II. $\mathcal{C}$-categories, $\mathcal{C}$-functors and $\mathcal{C}$-morphisms, Publ. Math. Debrecen 24 (1977), 351-361. MR 58:16834a

[15] Peter Schauenburg, Hopf modules and the double of a quasi-Hopf algebra, Trans. Amer. Math. Soc. 354 (2002), 3349-3378. MR 2003c:16050

Mathematisches Institut, Universität München, Theresienstr. 39, 80333 München, Germany

E-mail address: schauen@rz.mathematik.uni-muenchen.de 